\title{
Parents who received transfer preparation had lower anxiety about their children's transfer from the paediatric intensive care unit to a general paediatric ward
}

Bouvé LR, Rozmus CL, Giordano P. Preparing parents for their child's transfer from the PICU to the pediatric floor. Appl Nurs Res 1999 Aug;12:114-20.

QUESTION: What is the effectiveness of transfer preparation to reduce the anxiety level of parents of children facing imminent transfer from a paediatric intensive care unit (PICU) to a general paediatric ward?

\section{Design}

Randomised \{allocation concealed $\}^{*}$, unblinded, controlled trial.

\section{Setting}

A tertiary medical centre in southeastern USA.

\section{Participants}

50 parents (mean age 31 y, age range 15-52 y, 62\% women, $80 \%$ white, $78 \%$ married) of children (mean age $3 \mathrm{y}$, age range 1 wk to $12 \mathrm{y}$ ) who were in a PICU (mean duration of PICU stay $5 \mathrm{~d}$ ). Inclusion criteria were first experience of a child in the PICU, child in hospital because of an acute illness (eg, trauma, surgical procedure, or diagnosis of disease), parent attendance in the PICU for $\geqslant 3$ hours/day, and child to be transferred out of the PICU to a general paediatric ward with $\geqslant 24$ hours of notice.

\section{Intervention}

Parents were allocated to an experimental $(\mathrm{n}=31)$ or control $(n=19)$ group. Parents in the intervention group were given a transfer preparation letter and a 10 minute verbal explanation by a nurse of the expected changes in the level of care by the ward staff and positive aspects of the transfer, and were encouraged to ask questions. Parents in the control group did not receive a letter or verbal explanation and were informed of the transfer immediately before it occurred (standard procedure).

\section{Main outcome measures}

Parental self reported anxiety levels were measured using Spielberger's State-Trait Anxiety Inventory (STAI) 24-48 hours before the child's transfer (pre-intervention score) and after the intervention 1-2 hours before the child's transfer (post-intervention score). The STAI consists of 2 separate scales, the state anxiety and trait anxiety scales; scores range between 20 and 80 and higher scores represent higher anxiety levels.

\section{Main results}

Mean pre-intervention STAI scores did not differ for intervention and control groups (state anxiety scores 45 and 47 , respectively, and trait anxiety scores 35 and 38 , respectively). Mean post-intervention STAI state anxiety scores were lower for the intervention than the control group (37 $v 47$ ) after controlling for pre-intervention trait anxiety $(\mathrm{p}<0.001)$ and pre-intervention state anxiety $(\mathrm{p}<0.001)$. Mean post-intervention STAI trait anxiety scores did not differ for the intervention and control groups (33 $v 36)$.

\section{Conclusion}

Parents of children facing imminent transfer from a paediatric intensive care unit to a general paediatric ward who received transfer preparation had lower anxiety levels than those who received the standard procedure.

*Information provided by author.

\section{COMMENTARY}

Several studies have described the stresses and responses of parents during a child's hospital stay in a PICU ${ }^{1-3}$; however, none has focused on parents' anxiety when their child is transferred out of the PICU onto a general paediatric ward. This study by Bouvé $e t a l$ is unique in that it tested a creative, cost effective, brief intervention aimed at helping to reduce parental transfer anxiety. A strength of the study is that it builds on the Lazarus Stress and Coping Model, a well known conceptual model. The sample size ensured adequate power, the participants were randomly assigned, and the outcome (state anxiety) was measured using an appropriate and valid tool.

There are several limitations to the study. It would have strengthened the description of the design if the authors had described how they randomised participants into the experimental and control groups. State anxiety was measured 1-2 hours before the child's transfer. The study would have been strengthened if data were also collected within 24 hours after the transfer. Because these data were not obtained, we do not know if the intervention reduced parental anxiety after the transfer had occurred.

The results are important to nurses who work in paediatric inpatient units. This intervention, which is easy and cost effective to implement, was effective in reducing immediate transfer anxiety in parents. Nurses working in PICUs should work with their colleagues on the regular paediatric ward to develop a similar intervention to help parents to cope with their child's transfer from the PICU. Margaret $\mathrm{S}$ Miles, RN, PhD, FAAN Professor, School of Nursing University of North Carolina at Chapel Hill Chapel Hill, North Carolina, USA

1 Curley MA, Wallace J. Effects of the nursing mutual participation model of care on parental stress in the pediatric intensive care unit-a replication. J Pediatr Nurs 1992;7:377-85.

2 Fisher MD. Identified needs of parents in a pediatric intensive care unit. Crit Care Nurse 1994;14:82-90.

3 Youngblut JM, Shiao SY. Child and family reactions during and after pediatric ICU hospitalization: a pilot study. Heart Lung 1993;22:46-54. 